Regular Article

\title{
Antifungal versus antibacterial defence of insect wings
}

\section{Elena P. Ivanova a,b,c,1, Denver P. Linklater ${ }^{\mathrm{a}, \mathrm{d}, 1}$, Arturo Aburto-Medina ${ }^{\mathrm{a}}$, Phuc Le ${ }^{\mathrm{a}}$, Vladimir A. Baullin ${ }^{\mathrm{c}}$,} Huu Khuong Duy Nguyen ${ }^{a}$, Roger Curtain ${ }^{e}$, Eric Hanssen ${ }^{e}$, Gediminas Gervinskas ${ }^{f}$, Soon Hock Ng ${ }^{d}$, Vi Khanh Truong ${ }^{a}$, Pere Luque ${ }^{\mathrm{g}}$, Georg Ramm ${ }^{\mathrm{f}}$, Han A.B. Wösten ${ }^{\mathrm{i}}$, Russell J. Crawford ${ }^{\mathrm{a}}$, Saulius Juodkazis ${ }^{\mathrm{d}}$, Shane Maclaughlin ${ }^{\mathrm{b}, \mathrm{j}}$

a School of Science, College of Science, Engineering and Health, RMIT University, Melbourne, Victoria 3001, Australia

${ }^{\mathrm{b}}$ ARC Research Hub for Australian Steel Manufacturing Melbourne, Victoria 3001, Australia

${ }^{\mathrm{c}}$ ARC Training Centre in Surface Engineering for Advanced Materials (SEAM), Swinburne University of Technology, Hawthorn, Victoria 3122, Australia

${ }^{d}$ Optical Sciences Centre, School of Science, Swinburne University of Technology, Hawthorn, Victoria 3122, Australia

e Ian Holmes Imaging Center, Bio21, University of Melbourne, Parkville, Victoria, Australia

${ }^{\mathrm{f}}$ Monash Ramaciotti Cryo EM Facility, 15 Innovation Walk, Clayton, Victoria 3168, Australia

${ }^{g}$ Museu de les Terres de l'Ebre, Gran Capità, 34, 43870 Amposta, Spain

${ }^{\mathrm{h}}$ Departament de Química Física i Inorgànica, Universitat Rovira I Virgili, 26 Av. dels Paisos Catalans, 43007 Tarragona, Spain

i Department of Microbiology, Utrecht University, Padualaan 8, 3584 CH Utrecht, the Netherlands

${ }^{\mathrm{j}}$ BlueScope Steel Products, Port Kembla, New South Wales, Australia

\section{H I G H L I G H T S}

- We present a novel concept of dual defence against surface colonisation of insect wings by bacteria and fungi.

- The antifouling and antibacterial properties of insect wings can be attributed to the unique combination of nanoscale structures on the wing surface.

- The robust fungal-repelling properties of damselfly wing surfaces were demonstrated to arise from the presence of entrapped air, facilitated by the surface nano-topography.

\section{A R T I C L E I N F O}

\section{Article history:}

Received 17 May 2021

Revised 11 June 2021

Accepted 14 June 2021

Available online 18 June 2021

\section{Keywords:}

Antibacterial

Antifungal

Self-cleaning

Superhydrophobic
G R A P H I C A L A B S T R A C T

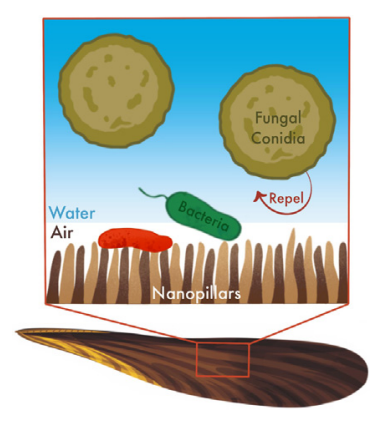

\begin{abstract}
A B S T R A C T
Hypothesis: The ability exhibited by insect wings to resist microbial infestation is a unique feature developed over 400 million years of evolution in response to lifestyle and environmental pressures. The selfcleaning and antimicrobial properties of insect wings may be attributed to the unique combination of nanoscale structures found on the wing surface.

Experiments: In this study, we characterised the wetting characteristics of superhydrophobic damselfly Calopteryx haemorrhoidalis wings. We revealed the details of air entrapment at the micro- and nano scales on damselfly wing surfaces using a combination of spectroscopic and electron microscopic techniques. Cryo-focused-ion-beam scanning electron microscopy was used to directly observe fungal spores and conidia that were unable to cross the air-liquid interface. By contrast, bacterial cells were able to cross the air-water interface to be ruptured upon attachment to the nanopillar surface. The robustness of the air entrapment, and thus the wing antifungal behaviour, was demonstrated after 1-week of water immersion. A newly developed wetting model confirmed the strict Cassie-Baxter wetting regime when damselfly wings are immersed in water.
\end{abstract}

\footnotetext{
1 These authors contributed equally to this work

E-mail address: elena.ivanova@rmit.edu.au (E.P. Ivanova)
} 
Findings: We provide evidence that the surface nanopillar topography serves to resist both fungal and bacterial attachment via a dual action: repulsion of fungal conidia while simultaneously killing bacterial cells upon direct contact. These findings will play an important role in guiding the fabrication of biomimetic, anti-fouling surfaces that exhibit both bactericidal and anti-fungal properties.

(c) 2021 Elsevier Inc. All rights reserved.

\section{Introduction}

Lifestyle and environmental pressures determine the unique features of the wing topography in flying insects [1,2]. Largewinged insects require self-cleaning wings with reduced wettability to decrease the consumption of inertial power during flight take-off. Their surface characteristics can be correlated to their ability to resist contamination and prevention of wetting of the wing surfaces [2]. This is especially important, since largewinged insects such as dragonflies, damselflies and cicadae cannot clean their wings by any physical manner. As such, insect wings have inspired investigation into the principles and application of
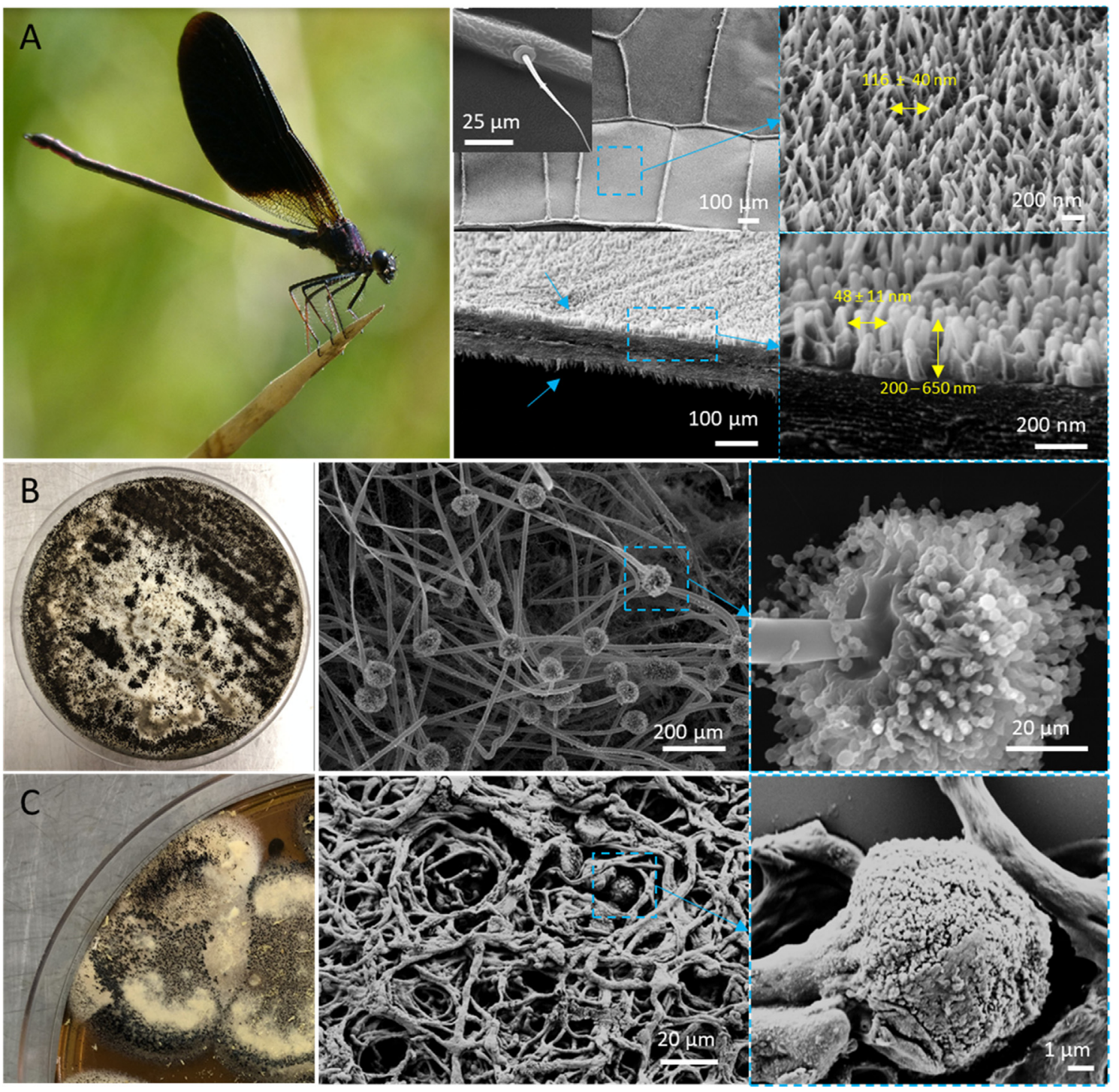

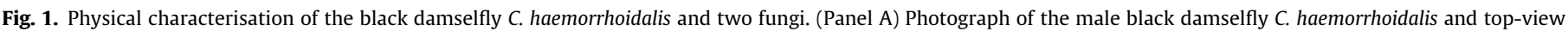

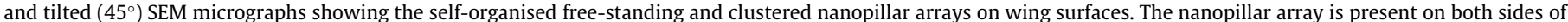

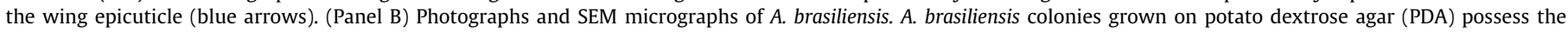

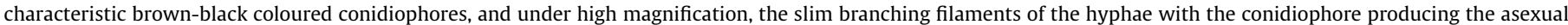

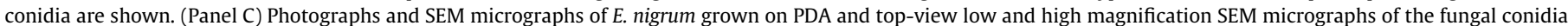

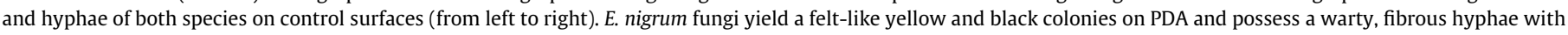

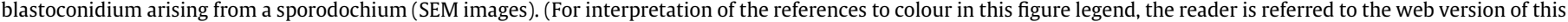
article.) 
aerodynamics, hydrodynamics, and importantly, self-cleaning effects of such surfaces [3]. Numerous biomimetic surfaces have been fabricated to take advantage of the naturally occurring selfcleaning properties of these topographies to achieve stable water-repellent, self-cleaning surfaces [4-6].

While investigating the antibacterial effects of insect wings, which have been proposed to resist bacterial attachment due to their superhydrophobicity, the nanopillar array on the surface of insect wings was found to play a role in the mechanical killing of bacterial cells that encounter the surface by the nanostructureinduced lysis [7-9]. It was initially observed for cicada wings immersed for an extended period $(24 \mathrm{~h})$ in bacterial suspension, that the wings were not able to effectively repel the contacting cells. All attached bacterial cells were found to be dead, ruptured by a later-reported mechano-bactericidal mechanism [9]. The wings of other insect species, including dragonflies and damselflies, also displayed substantial bactericidal activity $[3,7,8]$. While the antibacterial effects of insect wings have been thoroughly investigated $[3,7,8,10]$, their mechanism of defence against fungal contamination remains unclear.

Fungal contamination of surfaces leads to persistent problems in industry, transport, and everyday life $[11,12]$. They are known to colonize a wide range of surfaces, including paint coatings, cellulose-based materials, metals, and concrete structures [1315]. For example, fungal biofilms are responsible for the effects of corrosion and fouling on the fuel efficiency of ships [16]. For antibiofouling applications, robust superhydrophobicity seems to be crucial for maintaining a continuous ability to repel fungi from the surface. Recently, there has been significant interest in surfaces that do not just exhibit 'slip' behaviour, where water condensation or droplets can slide off a surface, but in surfaces that also remain superhydrophobic when fully immersed in water, i.e., for application in microfluidic systems $[17,18]$. For example, Lee \& Hwang recently fabricated a superhydrophobic aluminium surface that could reduce fungal contamination of industrial brazed aluminium heat exchangers [19]. The superhydrophobic surface was generated by the growth of hierarchical micro-nanostructures and the subsequent application of a hydrophobic polymer coating. Although fungal colonisation occurred on the treated heat exchangers and non-treated BAHEs in the same manner, the presence of surface superhydrophobicity, based on a combination of surface architecture and chemistry, allowed for the efficient selfcleaning of these substrates.

In the context of biomedical applications, including implantable devices, numerous different types of antibacterial surfaces have been developed [20-22], yet filamentous fungi are also an important, but often overlooked, component of hospital acquired infections [23]. The incident of serious, life-threatening fungal infections has increased substantially in recent years. Almost a decade ago, an editorial published in the journal Science aimed to lift awareness of the scale of the threat to human health posed by fungi, and the urgent need to find new avenues for the treatment and prevention of fungal disease [24].

Therefore, the aim of this work was to understand the mechanism by which insect wings can prevent fungal colonisation. Herein, we assessed the antifungal properties of the wings of the damselfly Calopteryx haemorrhoidalis using two species of filamentous fungi, Aspergillus brasiliensis ATCC 9642 and Epicoccum nigrum ATCC 9348. Analyses of optical images and scanning electron microscopy (SEM) micrographs were used to characterise the interactions of fungal conidia and the damselfly wings used in this study (Fig. 1). The surface wetting behaviour and the antifungal properties of the wing surfaces were investigated using advanced imaging techniques, such as cryo-SEM and cryo-focused-ionbeam (FIB) milling, to visualise the air-water-nanopillar interface and the water-air-nanopillar-fungi biointerface. Here, we present the first demonstration of the role of the wing nanopillar topography in resisting fungal conidia attachment.

\section{Materials and methods}

Chloroform $\geq 99 \%$ purity, anhydrous absolute ethanol $\geq 99.0 \%$ purity were purchased from Merck (Merck Pty Ltd., Australia). $25 \%$ glutaraldehyde grade I, in $\mathrm{H}_{2} \mathrm{O}$, specially purified for use as an electron microscopy fixative was purchased from Sigma Aldrich (Sigma-Aldrich Pty Ltd, Australia).

\subsection{Damselfly wing preparation}

C. haemorrhoidalis is a species of damselfly that is known to inhabit clear European streams and rivers in the Western Mediterranean area [25]. For distinction purposes, the wings of mature male damselfly of the species $C$. haemorrhoidalis are dark brown, while the female damselfly wings are lightly coloured and translucent. Mature male $C$. haemorrhoidalis damselfly specimens were collected from the Els Ports region situated at the borders of Catalonia, Valencia, and Aragon. The membrane of the damselfly wings was dissected into square sections on the dorsal side of the forewing, approximately $5 \times 5 \mathrm{~mm}^{2}$ in size, using a surgical blade. The wing samples were then briefly rinsed with Milli-Q $\mathrm{H}_{2} \mathrm{O}$ (resistivity of $18.2 \mathrm{M} \Omega \mathrm{cm}^{-1}$, Millipore, U.S.A.) and finally blow-dried using $99.99 \%$ purity nitrogen gas, as described elsewhere [26].

The wings were de-waxed by immersing them in chloroform for $30 \mathrm{~min}$ and then rinsing thoroughly with Milli- $\mathrm{Q}^{\circledR}$ water. Wings were damaged by pressing the surfaces with a blunt object to flatten the pillars.

\subsection{Fungal growth}

Two fungal species were used in this study, A. brasiliensis ATCC 9642 and E. nigrum ATCC 9348. A. brasiliensis and E. nigrum both grow aerobically on organic matter and can survive across a wide temperature range of $6-47^{\circ} \mathrm{C}$ [27] (Figure S1). These strains were purchased from American Type Culture Collection (ATCC, Manassas, Virginia, USA). The fungi were grown on potato dextrose agar (PDA, BD Difco ${ }^{\mathrm{TM}}$, Thermo Fischer Scientific, Waltham, Massachusetts, USA), supplemented by fine particles of wood chips. Potato dextrose agar plates were incubated at $25^{\circ} \mathrm{C}$ in dark conditions for 10 - 14 days, and aerial conidia were harvested by flooding a plate with sterile Milli- $Q^{\circledR}$ water. Conidia suspensions were filtered through a single layer of Micracloth ${ }^{\mathrm{TM}}$ (Merck Pty Ltd., Australia), and spore concentrations were determined by direct count using a haemocytometer. The desired concentration was diluted to obtain $1 \times 10^{6}$ cells $\mathrm{mL}^{-1}$.

Solutions of fungal conidia suspensions in Milli- $Q^{\circledR}$ water (1$3 \times 10^{6}$ cells $\mathrm{mL}^{-1}$ ) were used to assess the adhesion of fungal conidia to damselfly wing surfaces. Wing surfaces with dimensions of $0.5 \times 0.5 \mathrm{~cm}^{2}$ were placed in sterile 24-well plates (Thermo Fischer Scientific, Waltham, Massachusetts, USA), and then submersed in $3 \mathrm{~mL}$ of $A$. brasiliensis and $E$. nigrum conidia suspensions, respectively. Intact damselfly wings and control surfaces were incubated at $25^{\circ} \mathrm{C}$ in dark conditions for periods of 1 , 3,7 and 14 days. Conidia attachment and fungal growth were compared between intact, de-waxed and damaged (scratched) damselfly wings.

After incubation, samples were removed and washed $3 \times$ with $1 \mathrm{~mL}$ of MilliQ ${ }^{\circledR} \mathrm{H}_{2} \mathrm{O}$. Wing surfaces were then fixed with $2.5 \%$ glutaraldehyde for $40 \mathrm{~min}$. Following fixation, the wings were submerged in MilliQ ${ }^{\circledR} \mathrm{H}_{2} \mathrm{O}$ and frozen at $-20^{\circ}$ overnight. The frozen wing samples were then lyophilised (Freeze Dryer Alpha 1-2 
LDPlus, CHRIST) and sputtered with a thin film of gold for SEM imaging.

\subsection{Wettability}

The surface wettability of the wings and the fungal conidia was examined by measuring the water contact angle (WCA), using the sessile drop method, at the point prior to droplet elongation as the tip was drawn away from the surface. The contact angle measurements were carried out in air using an FTA1000c equipped with a nanodispenser (First Ten Ångstroms, Inc., Portsmouth, VA, USA). Seventeen measurements were taken across the entire surface of each damselfly wing and directly on the fungal colonies (Figure S2).

\subsection{Bacterial growth}

Pseudomonas aeruginosa ATCC 9721 was purchased from the American Type Culture Collection (ATCC) and stored at $-80^{\circ} \mathrm{C}$ until required. Bacteria were refreshed on nutrient agar (Oxoid, Thermo Fisher Scientific Australia Pty Ltd, Australia) for $24 \mathrm{~h}$ at $37^{\circ} \mathrm{C}$ and then sub-cultured a further two times prior to SEM and confocal laser scanning microscopy experiments.

\subsection{Confocal laser scanning microscopy (CLSM)}

C. haemorrhoidalis damselfly wings were incubated for $24 \mathrm{~h}$ with a suspension of $P$. aeruginosa ATCC 9721 in phosphate buffered saline (PBS, $\mathrm{pH} 7.4$ ) at $25^{\circ} \mathrm{C}$. Bacterial suspensions were prepared by dispersing 1 loopful of bacteria in PBS and diluting until an optical density $\left(\mathrm{OD}_{600 \mathrm{~nm}}\right)$ of 0.1 was achieved using a Halo RB-10 UV-VIS spectrophotometer (Dynamica Scientific Ltd, U.K.). Whole wings were mounted on the bottom of a $35 \mathrm{~mm}$ glassbottomed Petri dish using Kapton@ tape (ProSciTech Pty Ltd, Australia) and $3 \mathrm{~mL}$ of the bacterial suspension was used to cover the entire wing. Prior to CLSM, the bacterial suspension was removed, and the attached bacteria were dyed using a LIVE/DEAD BacLight Bacterial Viability Kit (Invitrogen, Molecular Probes, U.S.A.) (including SYTO $9^{\circledR}$ and propidium iodide). The wing samples were then rinsed and re-submerged in $3 \mathrm{~mL}$ of PBS and viewed using ZEISS LSM 880 Airyscan upright microscope (Carl Zeiss Microscopy, Germany) in reflectance imaging mode with a $20 \times$ Planapochromat water immersion objective.

\subsection{Cryo-SEM}

Prior to the cryo-SEM imaging, insect wings submerged in water for 1-day and 1-week, respectively, were attached to a SEM copper stage using a Tissue-Tek glue (Sakura Finetek, Alphen aan den Rijn, The Netherlands). The wetted wings were then manually plunge-frozen into liquid N2 and transferred into the cryostage pre-chamber, Alto 2500 (Gatan, U.K.) at $-140{ }^{\circ} \mathrm{C}$, before being sublimed at $-95{ }^{\circ} \mathrm{C}$ for $3 \mathrm{~min}$ to remove free water molecules. Samples were then coated with $5 \mathrm{~nm} \mathrm{Au} / \mathrm{Pd}$ at $-140{ }^{\circ} \mathrm{C}$. After $\mathrm{Au} / \mathrm{Pd}$ coatings, samples were transferred into the SEM stage at $-140^{\circ} \mathrm{C}$. Samples were then imaged using Quanta 200F SEM (FEI, Hillsboro, OR) at $10 \mathrm{kV}$.

The water condensation experiments were also carried out using the Quanta 200F SEM. The electron beam energy and current of $5 \mathrm{keV}$ and $0.019 \mathrm{nA}$, respectively, were used. Water condensation was achieved by decreasing the sample temperature below the saturation temperature $0-10{ }^{\circ} \mathrm{C}$ corresponding to the environmentalSEM (ESEM) chamber vapor pressure of 700-1300 Pa (5-10 Torr). The condensing droplets were imaged every $\sim 1 \mathrm{~s}$ and corresponding images were captured with a $1 \mathrm{~Hz}$ frequency.

\subsection{Cryo-FIB-SEM}

For cryo-preservation, damselfly wings were trimmed to a size of 1-2 $\mathrm{mm}^{2}$ and clamped between a double grid (Oyster) GD50/50 (EMS, U.S.A.). The specimens were placed into a Vitrobot Mk IV chamber (FEI, US) set to $100 \%$ humidity and $25{ }^{\circ} \mathrm{C}$ and after a 1 minute incubation, automatically plunged into a reservoir with liquid ethane. The frozen insect wings were then transferred into liquid nitrogen and to a sample cryo-manipulation system (EM VCM, Leica Microsystems, Germany), and kept submerged in liquid nitrogen. The specimen was moved to the high vacuum coater (EM ACE600, Leica Microsystems, Germany) using a vacuum cryo-transfer system (EM VCT500, Leica Microsystems, Germany). The insect wings were then e-beam coated with $\mathrm{a} \sim 10 \mathrm{~nm}$ thick layer of platinum, to prevent water ice charging-up during the later SEM imaging and ion-beam milling stages. The coated specimen was transferred to a FIB-SEM (Helios G4 UX, Thermo Fisher Scientific (FEI), U.S.A.) equipped with a custom built cryo-stage (Leica Microsystems, Germany) using the VCT500. Condensed water droplets were imaged under high vacuum using cryo-SEM at $1-5 \mathrm{kV}$ using the secondary electron (SE) detector in immersion mode while FIB set to $30 \mathrm{kV}$ was used to mill cross-sections, in order to reveal the interfaces between water droplets and the insect wing surface.

\subsection{Raman spectroscopy}

Detection of air entrapment at nano- and micro-scale was performed using a Raman micro-spectrometer (WiTEC, Ulm, Germany) with a laser wavelength of $532 \mathrm{~nm}(\mathrm{hv}=2.33 \mathrm{eV})$. A water-immersion $60 \times$ magnification objective lens (numerical aperture $=0.9$ ) was employed to map the water distribution over the nanopillar array. The displacement of the objective lens between the focal plane of the wing surface and the water layer, which were based on the characteristic peak intensity for $\mathrm{C}-\mathrm{C}$ bonds (aromatic rings in melanin) and $\mathrm{O}-\mathrm{H}$ bonds (covalent bonds in water), was used to estimate the thickness of the air blanket. A grid of 100 spectra $\times 100$ spectra was acquired for a $10 \times 5 \mu \mathrm{m}^{2}$ scanning area, with an integration time of $1 \mathrm{~s}$ per spectrum. Scanning was performed on three different areas of every sample immediately after initial water-immersion at room temperature.

\subsection{SEM}

High-resolution SEM images of native wing surfaces, fungal conidia, and wing surfaces with attached conidia were obtained at 20,000,60,000, and 100,000 magnifications using the SEM capabilities of the electron beam lithography tool (RAITH150 Two, Raith $\mathrm{GmBH}$, Germany) at $5 \mathrm{kV}$. Wing samples were coated with thin gold films of $7.5 \mathrm{~nm}$ in thickness using a MP-19020NCTR NeoCoater (JEOL Ltd, Japan), before viewing with the microscope. The height of nanopillars was determined by tilting the samples at a $45^{\circ}$ angle.

Nanopillar width and tip diameters were measured manually using Image software (version 1.50i), an automated approach was found to be unsuitable because of the natural bending and clustering of the nanopillars. To obtain pillar density measurements using ImageJ, the colour threshold for binary SEM images (Figure S4) was adjusted and the particles were analysed. Particles with a diameter below $30 \mathrm{~nm}$ were removed as artefacts. The nanofeature density, inclusive of free-standing and clustering nanopillars, was determined using measurements taken over six regions, $1.5 \mu \mathrm{m} \times 2 \mu \mathrm{m}$ in area, on each of the wing surfaces of the damselfly. 


\subsection{Bouncing droplet behaviour}

Bouncing water droplets were studied using a Phantom v2512 high-speed camera at $28,000 \mathrm{fps}$ and $768 \times 768$ resolution. Droplets were released $13 \mathrm{~mm}$ above the wing surfaces at room temperature using an FTA1000c equipped with a nanodispenser (First Ten Ångstroms, Inc., U.S.A.). The water droplet volume used was $9.6 \pm 0.8 \mu \mathrm{L}$, with a diameter of approximately $2.6 \pm 0.08 \mathrm{~mm}$. The droplet bouncing behaviour was characterized as 'pancake' or conventional bouncing. This was determined by calculating the ratio (Q) between the lateral extension diameter of the droplet as it detached from the surface $\left(d_{\text {jump }}\right)$ and the maximum diameter of the water droplet on the surface $\left(d_{\max }\right)$, where $Q>0.8$ indicated a pancake bounce. The Weber number was determined for each sample using the equation $W e=\rho v^{2} r_{0} / \gamma$, where $\rho$ is the water density, assumed as $997 \mathrm{~kg} \mathrm{~m}^{-3}, v$ is the impact velocity, $r_{0}$ is the droplet radius $(1.3 \mathrm{~mm})$, and $\gamma$ is the surface tension of the droplet, accepted as $7.2 \times 10^{4} \mathrm{Nm}^{-1}$. For all our experiments, it was $9.26 \pm 0.27$.

\section{Results and discussion}

\subsection{Physico-chemical characterization of C. Haemorrhoidalis damselfly wings}

SEM was used to analyse the C. haemorrhaoidalis wing topography. The wing epicuticle was characterised by a disordered distribution of nanopillars with mean height of $433 \pm 71 \mathrm{~nm}$, diameters of $48 \pm 11 \mathrm{~nm}$ and interpillar spacing of $116 \pm 40 \mathrm{~nm}$. The $C$. haemorrhoidalis damselfly wings were found to be superhydrophobic, exhibiting apparent WCAs $\left(\theta^{*}\right)$ across the wing surface ranging from $155^{\circ}$ to $170^{\circ}$ (Fig. 2A). The superhydrophobicity of the wing surface may be attributed to the wing epicuticle structure (which possesses a nanopillar array) and the chemical composition of the epicuticle on the wing membrane [1], shown in Figure S3. Briefly, synchrotron-sourced infrared imaging of the C. haemorrhoidalis wings revealed the typical components of the surface wax. These included amide bands that are indicative of the protein and chitin components of the wing, and characteristic peaks corresponding to melanin (see Supplementary Results). The outer layer of the insect wings, known as the cuticulin, has a strong affinity for the oriented polar groups of the wax layer, therefore the non-polar ends of the long-chain hydrocarbons tend to orient away from the surface [28]. To determine the contribution of the epicuticle structure to the wing superhydrophobicity, de-waxed wings were used as the non-structured control surfaces since they lack any nanopillars on the wing membrane. The moderate hydrophobicity $\left(114 \pm 8^{\circ}\right)$ of the 'de-waxed' wings can only be attributed to the lipid content of the inner cuticle layers (chitin) following the 'stripping' of the outer cuticle and the nanopillar structures. This observation agrees with that reported in previously published data on the contact angle of flat chitin sheets [29].

Raman analysis (Fig. 2B) of water-immersed damselfly wings confirmed the presence of a large air cushion, as observed in the photograph in Fig. 2A. Peaks at 1380 and $1580 \mathrm{~cm}^{-1}$ indicate the D-band and G-band of carbon compounds, respectively, resulting from the combination of $\mathrm{N}-\mathrm{H}$ bending and $\mathrm{C}-\mathrm{N}$ stretching of melanin, attributed to the wing surface [30]. As the z-height was increased, all signals for $\mathrm{C}-\mathrm{C}$ bonds were lost (0 CCD counts), indicative of the air layer, before the peak corresponding to water ( $\mathrm{O}-\mathrm{H}$ bonds) at $3430 \mathrm{~cm}^{-1}$ appeared. The peak located at $3430 \mathrm{~cm}^{-1}$ is attributed to the Raman scattering of partly hydrogen-bonded water. The thickness of the air layer after initial submersion was measured to be approximately $300 \mu \mathrm{m}$. This air cushion was observable for more than two weeks, although it became noticeably thinner over this period (Figure S4).

\subsection{Assessment of antifungal behaviour of Calopteryx haemorrhoidalis wings}

The initial step in fungal fouling involves the attachment of the fungal conidia to the biotic or abiotic surface (Figure S1) [31]. Fungal conidia and aerial hyphae are encased by a hydrophobic protein coat [32,33] aiding their dispersal on the surface of water droplets [34]. Thus, fungal fouling is closely associated with wetting phenomena in the solid-water-biological matter system. The WCA, hydrophobicity index and zeta potential of $A$. brasiliensis and $E$. nigrum conidia, respectively, are shown in Figure S2 and Table S1. Here, we utilized the spores and conidia of $A$. brasiliensis and $E$. nigrum to investigate their interactions with damselfly wing surfaces during water submersion.

The dynamic interactions of environmental fungi E. nigrum and A. brasiliensis conidia with the damselfly wings were studied over the course of 1,3- and 7-days. Analyses of SEM images revealed that the conidia and spores of both fungal strains were unable to colonize the wing surfaces after 7-days incubation (Fig. 2C, Figure S5). By contrast, the de-waxed wings, used as control surfaces, were successfully colonized by fungal conidia and spores after just 1 day of incubation (Fig. 2C).

Direct contact between the wing surface and conidia, achieved by pressing the wing to the conidia lawn, resulted in widely varied attachment patterns (Figure S6). Intact wings (no visible surface damage) resisted colonization by $A$. brasiliensis spores whereas those wings with surface defects incurred 5.5-fold increase in attachment (Figure S6). This agrees with the observations made for the attachment of fungal conidia and spores to wing surfaces during liquid submersion. Thus, in both air and liquid submersion conditions, it is hypothesized that repulsion of fungal conidia/ spore attachment may stem from a combination of surface chemistry and surface topography where (in liquid conditions) air entrapment does not allow fungal contact with the wing membrane and dually, a reduction in the amount of 'favorable' surface area for attachment.

In contrast, after incubation of the wings in physiological suspension containing $P$. aeruginosa bacteria for 1 week, bacterial cells attached to the surface were detected but were observed to be mechanically lysed [8] (Fig. 3A). Bacteria were demonstrated to escape the liquid phase and cross the air-water interface and the thick air-cushion to successfully attach onto the wing surface (where they were subsequently ruptured and killed by the action of the surface nanopillars [9]). CLSM in reflectance imaging mode revealed the presence of dead bacteria (stained red by membrane impermeable propidium iodide (PI)) directly underneath the areas of the membrane covered with air after only $24 \mathrm{~h}$ of incubation in PBS (Fig. 3B). Thus, bacterial cells were observed to attach to the surface, and die, beneath layers of air, indicating that bacteria can cross the air-water interface. Air bubbles are observed in the merged bright field image being released from the surface.

When considering a colloidal suspension in an aqueous medium, the total forces interacting on a suspended colloid nearing the air-water interface are governed by van der Waals, electrostatic, and, hydrophobic forces [35]. Van der Waals forces are repulsive because the air-water interface is negatively charged. However, this electrostatic repulsion can be overcome due to the hydrophobic interactions taking place at the air-water interface. Thermodynamically, the air-water interface is an energetically favorable space for colloids in the micron-size range (such as bacteria) compared to other regions within the bulk of the suspension [36]. If the colloidal particle or air-water interface is in motion, additional inertial forces come into play [36]. If the net force inter- 
A

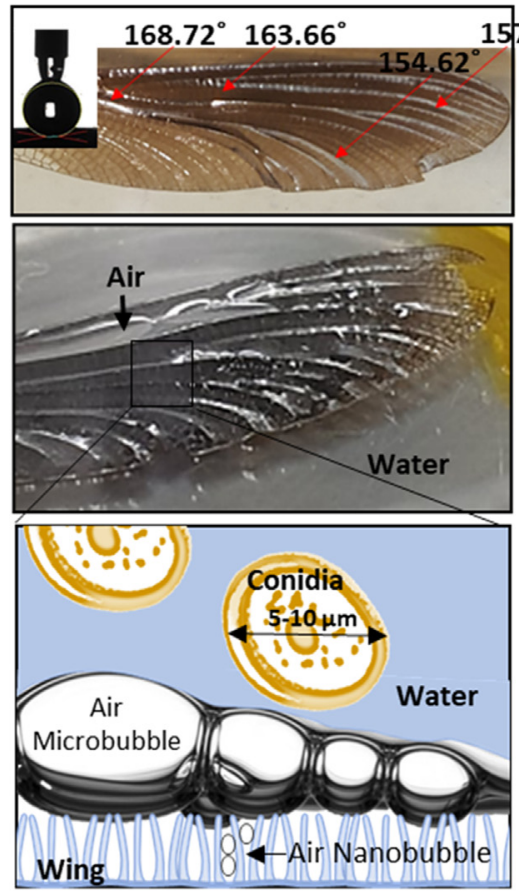

C

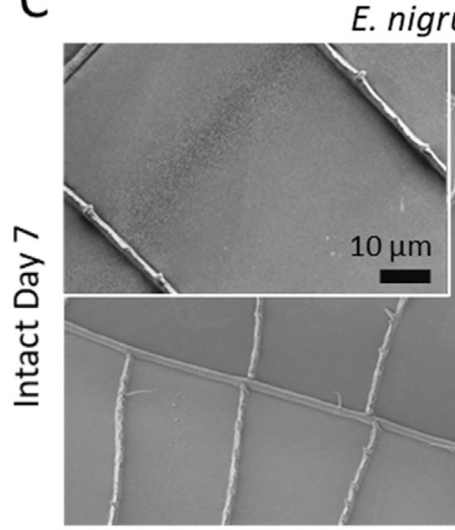

B $57.83^{\circ}$

3079 CCD cts

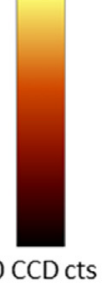

0 CCD cts
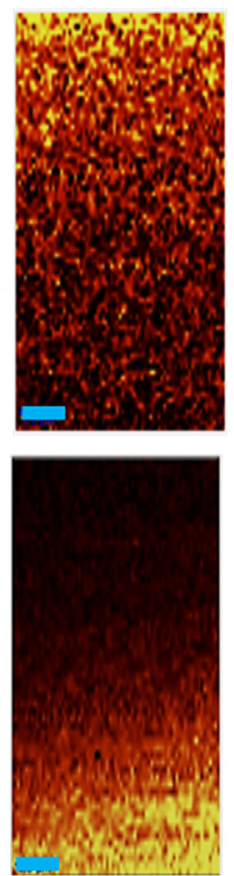

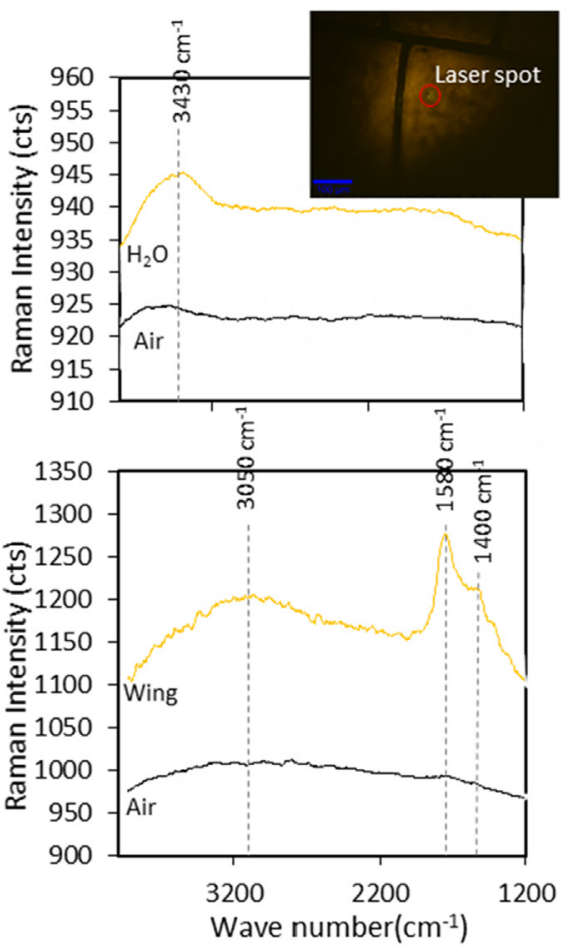

A. brasiliensis

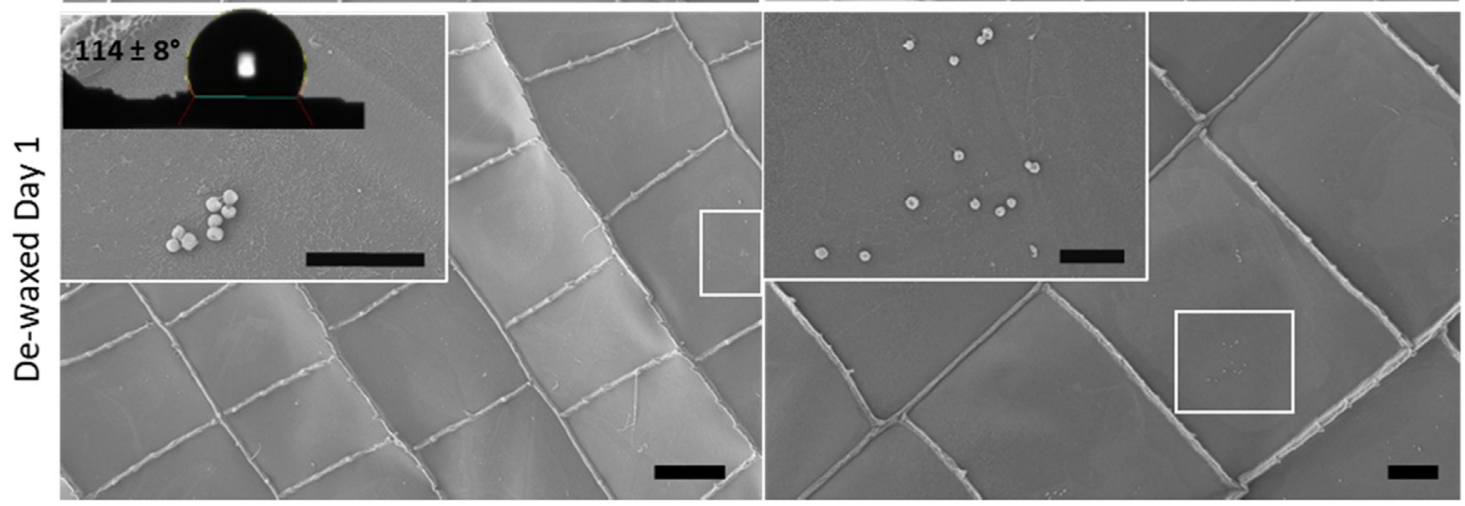

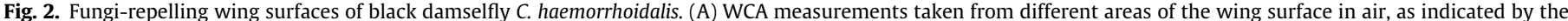

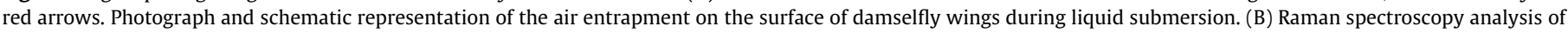

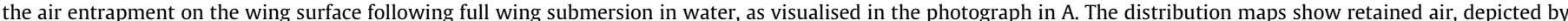

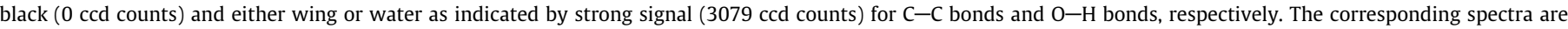

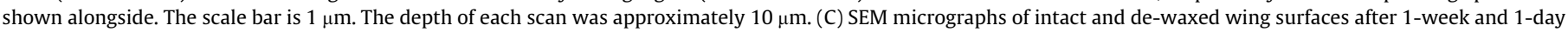

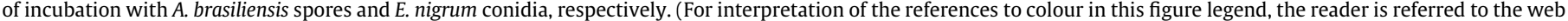
version of this article.) 

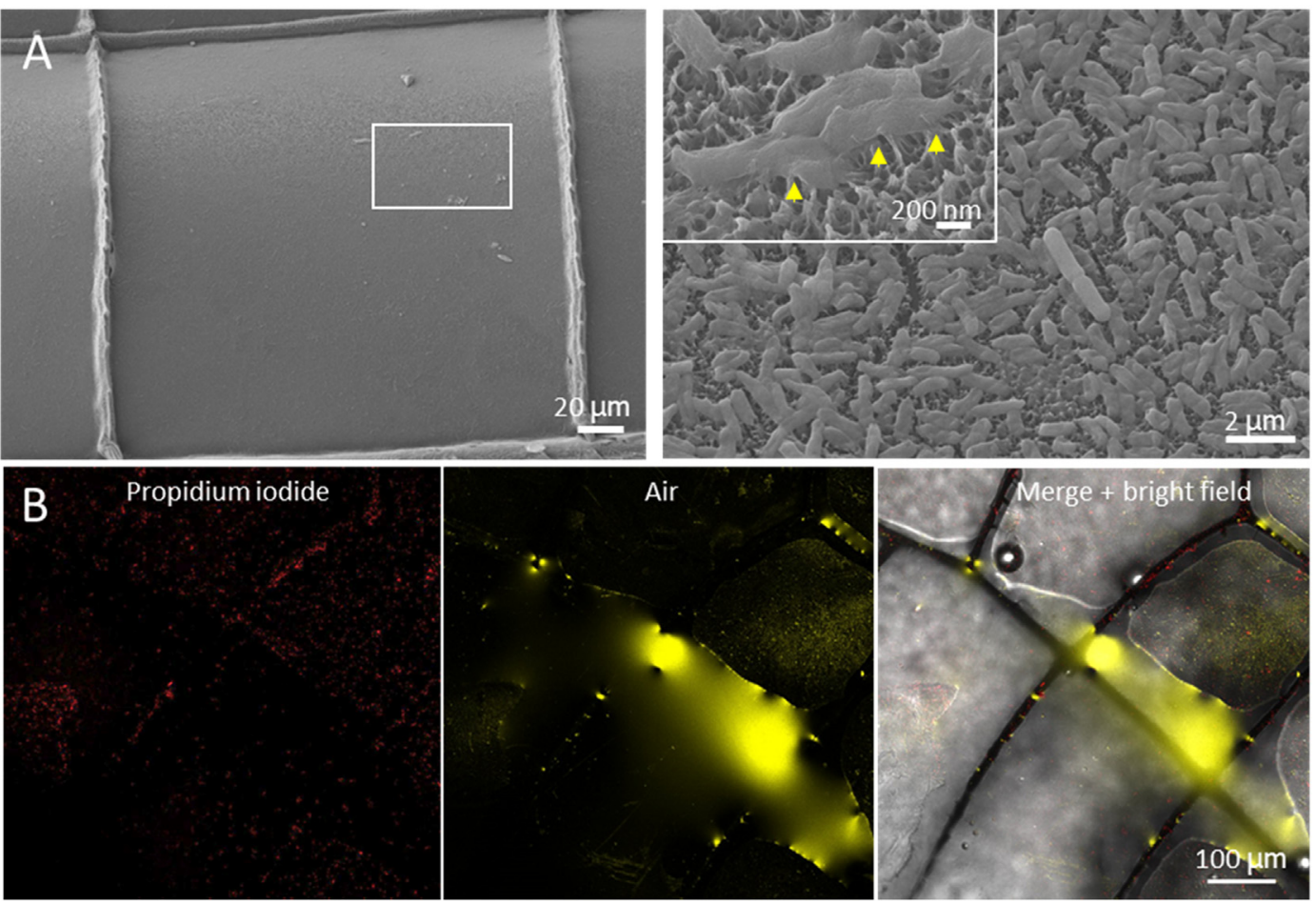

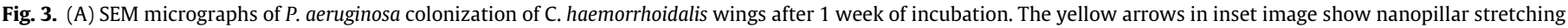

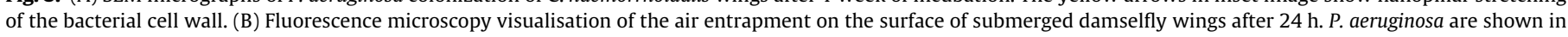

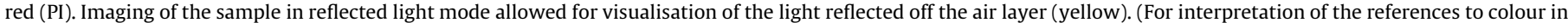
this figure legend, the reader is referred to the web version of this article.)

acting on a colloid approaching the air-water interface are positive, then the colloid will attach to and penetrate the interface, forming a three-phase contact line [37]. These principles also govern the interactions between bacteria and air-water interfaces $[38,39]$. Bacteria that collect at the air-water interface can be expected to alter the interfacial tension through the production of surface-active agents such as surfactants (low molecular weight amphipathic compounds) and may enable bacterial cells to land on the nanopillars.

To confirm and visualise the air layer between the nanoscale pillars on the wing surfaces and to assess the stability of the air entrapment, the wings were retained in water for 1 day and 1 week and then plunge-frozen for cryo-SEM (Fig. 4A, B). In Fig. 4A, no frozen liquid layer is visible on the wing surface following submersion in water for $24 \mathrm{~h}$. Thus, no liquid droplets can be retained on the surface upon removal from the liquid medium after water submersion for 1 day. However, after prolonged submersion in water (1 week) the air layer was reduced in volume and thickness; a frozen water layer can be observed covering the wing membranes, adhering to the intersecting veins (Fig. 4B). The layer of vitrified water was found to behave as a blanket lying atop a cushion of air. The 'breaks' in the water blanket (Fig. 4B inset) are most likely due to the variation in height distribution of the nanopillar array, where taller nanopillars facilitate air entrapment of varying thickness. Retention of the wing in an inoculating media of PBS containing both $P$. aeruginos a and fungal conidia for 1 week resulted in the observance of bacterial cells attached to the nanopillar topography, surrounded by the frozen water blanket (Fig. 4C); however, conidia attachment was not detected.

Investigation of fungal conidia attempting to land on the damselfly wing surfaces was also performed using cryo-SEM. Both $A$. brasiliensis and E. nigrum were found to be trapped at the liquidair interface, as shown in Fig. 4D and E, respectively. Therefore, the air-liquid interface is demonstrated to play an important role in inhibition of fungal fouling. Comparative analyses of fungal attachment to damaged (scratched) wing surfaces further confirmed the results obtained for dewaxed wing surfaces. In SEM images (Figure S4), the germination of the spores and conidia and production of hyphae was associated with hyphae being suspended above the wing surface, until contact is made only on a damaged section of the wing, where a surface scratch is obvious. These observations confirm that fungal conidia were physically unable to contact the wing membrane, unless there is some surface defect present that has removed some of the nanopillar array, or they can attach to the more hydrophilic areas of the wing, i.e., the veins.

Superhydrophobic surfaces can also cause water droplets, encountering the surface, to bounce. These droplets can undergo a phenomenon known as the 'pancake' bouncing [40]. Pancake bouncing occurs as droplets flatten and spread on impact with the surface before lifting off in a flattened pancake-like shape. Droplets are classified by the $Q$ ratio, which is the ratio between the lateral extension diameter of the droplet as it detaches from the surface $\left(d_{\text {jump }}\right)$ and the maximum diameter of water droplet on the surface $\left(d_{\max }\right)$. $Q>0.8$ indicates a pancake bounce [40]. Here, we analysed the bouncing droplet behaviour with and without fungal conidia being present on the wings of $C$. haemorrhoidalis, as shown in Fig. 5A, Figure S8). The total contact time of the bouncing droplet was found to be $2.0 \pm 1.1 \mathrm{~s}$ (Supplementary methods). The surface tension of a droplet can be changed by the presence of surface active amphiphilic compounds such as surfactants [41]. Therefore, the change in surface tension of a water droplet due to a high concentration of fungi was tested. However, no observable difference in contact time was able to be detected between the droplets with, or without, conidia. By contrast, the contact angle of both nutrient media and PBS solutions were substantially altered by bacterial growth at $37^{\circ}$ for $24 \mathrm{~h}$ that effectively reduced surface tension by approximately 5 times. Nutrient broth 

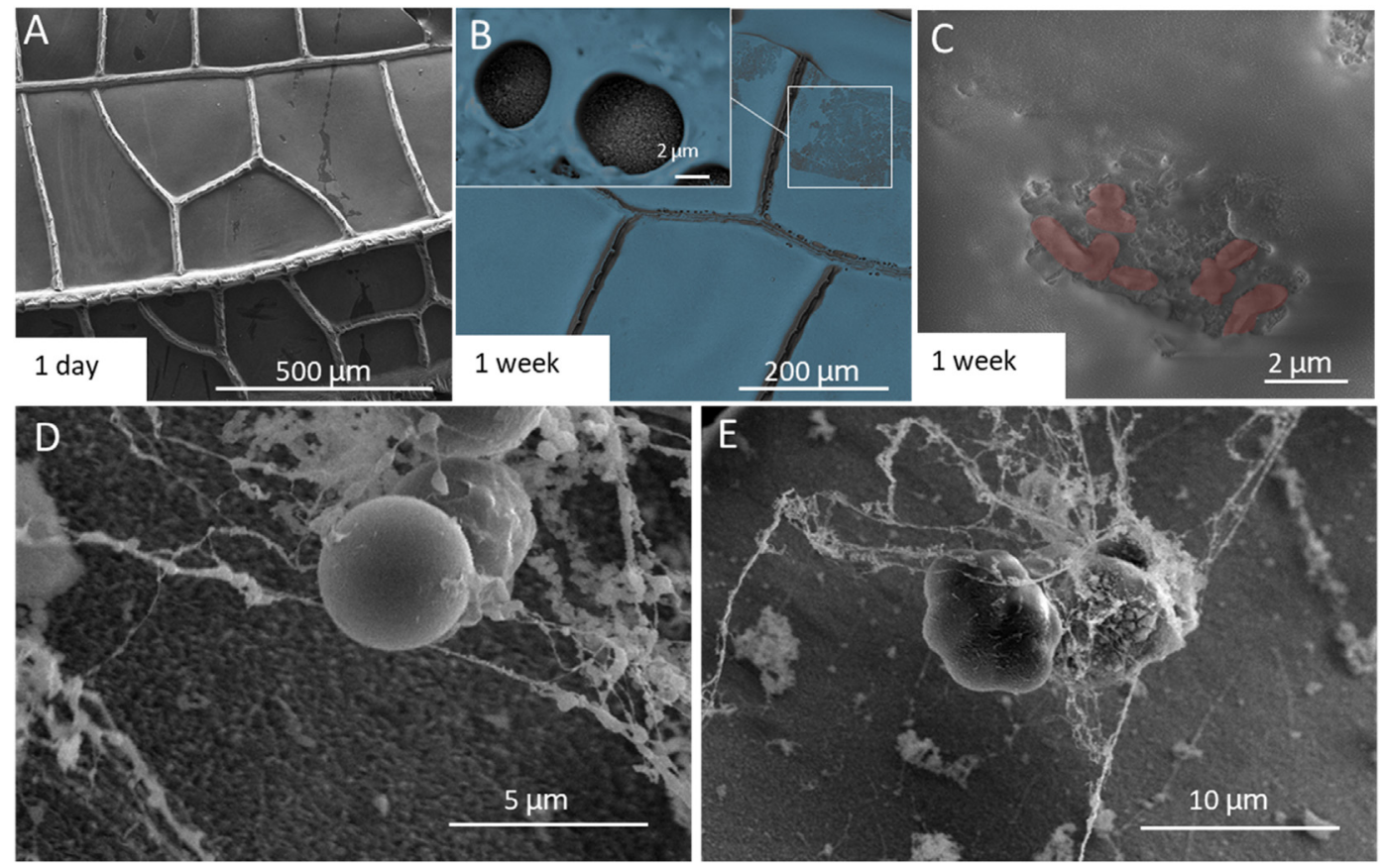

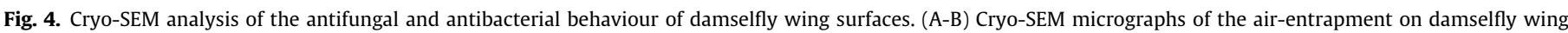

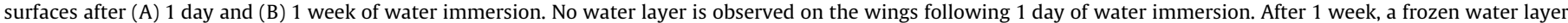

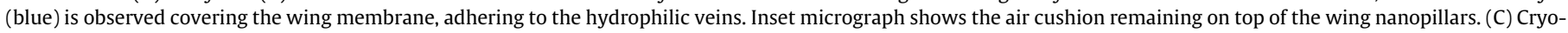

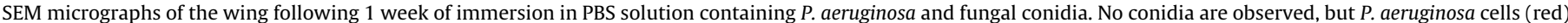

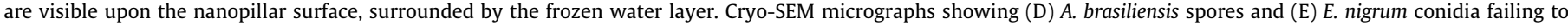

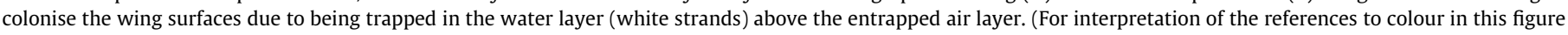
legend, the reader is referred to the web version of this article.)

and PBS solutions exhibited contact angles of $144 \pm 7^{\circ}$ and $161 \pm 6^{\circ}$, respectively on the wing surfaces. These values reduced to $98 \pm 5^{\circ}$ and $125 \pm 2^{\circ}$ for nutrient broth and PBS, respectively, in the presence of bacterial growth (Fig. 6G). Thus, these results confirm our hypothesis that bacteria collecting at the air-water interface on superhydrophobic surfaces can alter the interfacial tension; however, penetration and crossing of the air-water interface by the bacteria still requires expenditure of mechanical energy despite a decrease in interfacial tension, and the mechanism of such a cross-over requires further detailed investigation.

\subsection{The micro-nanoscale air-entrapment on the Calopteryx haemorrhoidalis wing}

The superhydrophobic properties of the damselfly wings could be further characterised using environmental SEM. Here, in situ imaging of micron-sized water droplets $(1-5 \mu \mathrm{m})$ condensing on the surfaces of the wings was observed (Fig. 5C). In general, there are four known stages of water condensation on any solid interface: nucleation, alignment, deformation and coalescence [42]. Water droplets nucleating on the surface of the wing membrane were observed to migrate towards the vein, where they aligned to form a three-phase contact line between the meniscus and the vein. Finally, the droplets were observed to deform and bridge by coalescence as they gathered more water and moved along the length of the vein. Water condensation was found to be inhibited on the epicuticle of the wing due to the presence of the nanopillar arrays that entrapped a consistent air layer, forcing the droplet to sit atop the pillars. These observations allowed us to assume that the veins of the wings, primarily made up of chitin and proteins, were more hydrophilic than the wing epicuticle itself and, therefore, act as the boundaries for the air pockets that lie atop the nanopillars (Fig. 5B).
The aircushion that sits atop the cuticle of insect wings can be easily visualised, which has enabled it to be observed as a homogenous layer sitting across the wing surface, yet the specific nanoscale geometry of the air-layer has yet to be revealed. Therefore, to visualise the air-entrapment between the nanopillars, water condensation on the wing surface was vitrified and the dropletnanopillar interface was investigated using Cryo-FIB-SEM (Fig. 6). As seen in Fig. 5C, condensing water droplets will preferentially migrate towards the hydrophilic wing veins; however, using this technique, the travelling water droplets could be captured in a frozen state on the wing membrane surface. Milling of the interface revealed the presence of air, observed as the dark contrast between the nanopillars on the wing membrane (Fig. 6B).

Assuming that the nanopillar pattern present on the surface of dragonfly wings is formed by cylindrical nanopillars with a height $H$, radius $r$ and interpillar distance $d$, we can estimate the Laplace pressure $\Delta p$ of air pockets trapped between pillars according to the original Cassie-Baxter model (Fig. 6D) [43]. The free energy of infinitesimal variation of height of the boundary between air and liquid is given by

$d F=-\Delta p d V+\gamma_{v l} d A_{v l}+\gamma_{s l} d A_{s l}-\gamma_{s v} d A_{s l}=0$

where the $\gamma_{v l}$ is the surface tension between air and liquid, $\gamma_{s l}$ is the interfacial tension between solid (pillar surface) and liquid and $\gamma_{s v}$ is the surface tension between solid and air; the $A_{\mathrm{vl}, \mathrm{sl}, \mathrm{sl}}$ are the surface areas of the vapour-liquid, liquid-solid and solid-liquid phases, respectively. Since the pillars are oriented perpendicularly to the surface, the corresponding area variations are expressed through the height variation parameter, $d h$. The volume variation $d V=\left(d^{2}-\pi r^{2}\right) d h$. The liquid-air interface does not change when the interface moves between pillars and thus $d A_{v l}=0$, The variation of the solid liquid interface $d A_{s l}=2 \pi r d h$. With this, the Laplace pressure in the air pocket can be expressed as 


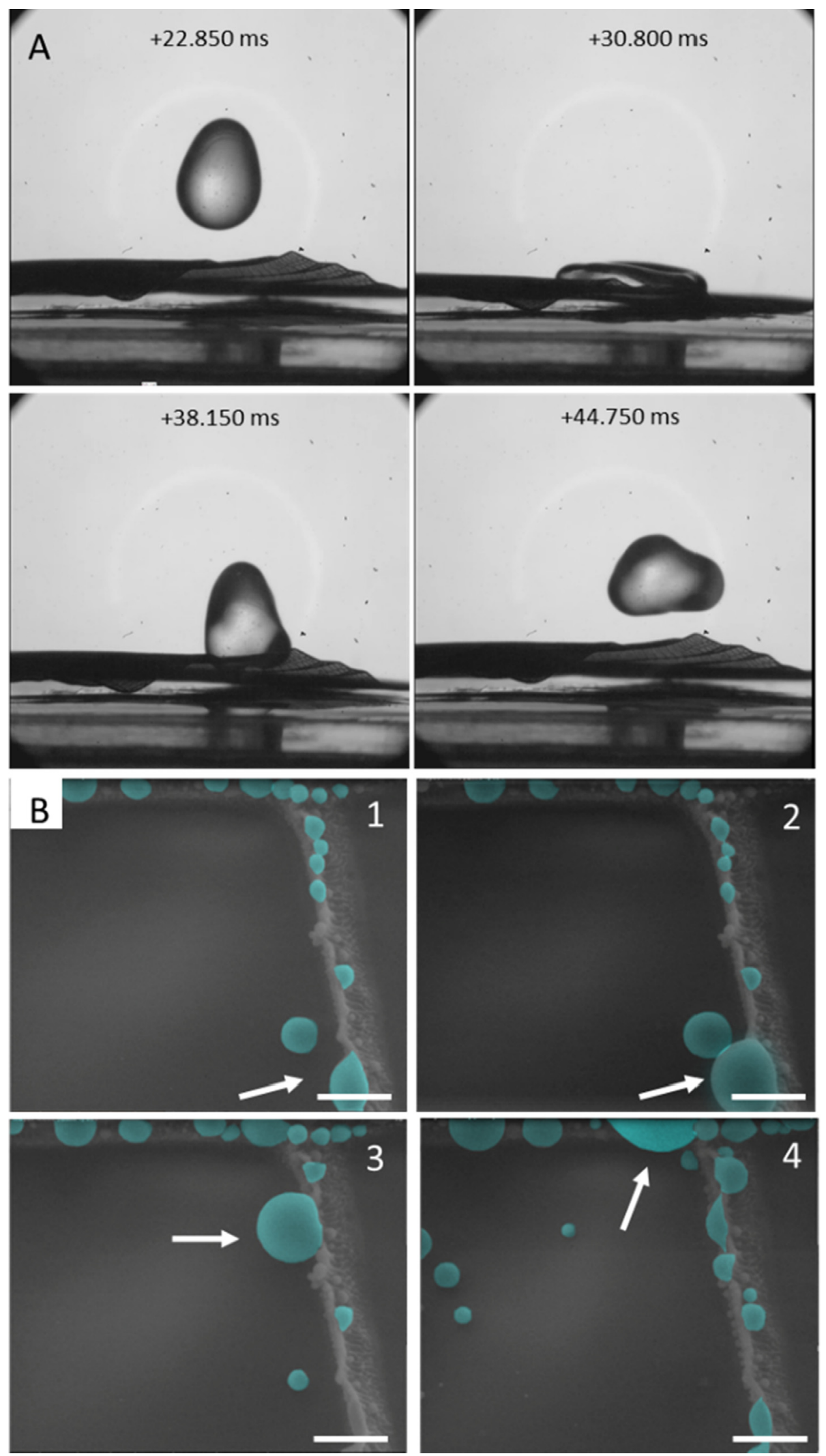

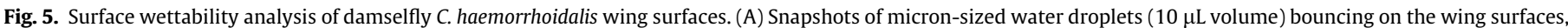

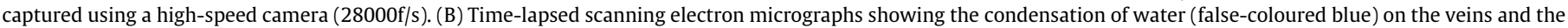

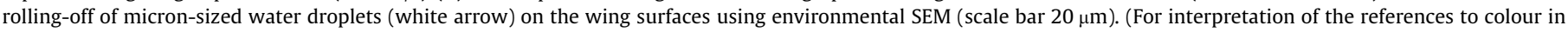
this figure legend, the reader is referred to the web version of this article.) 

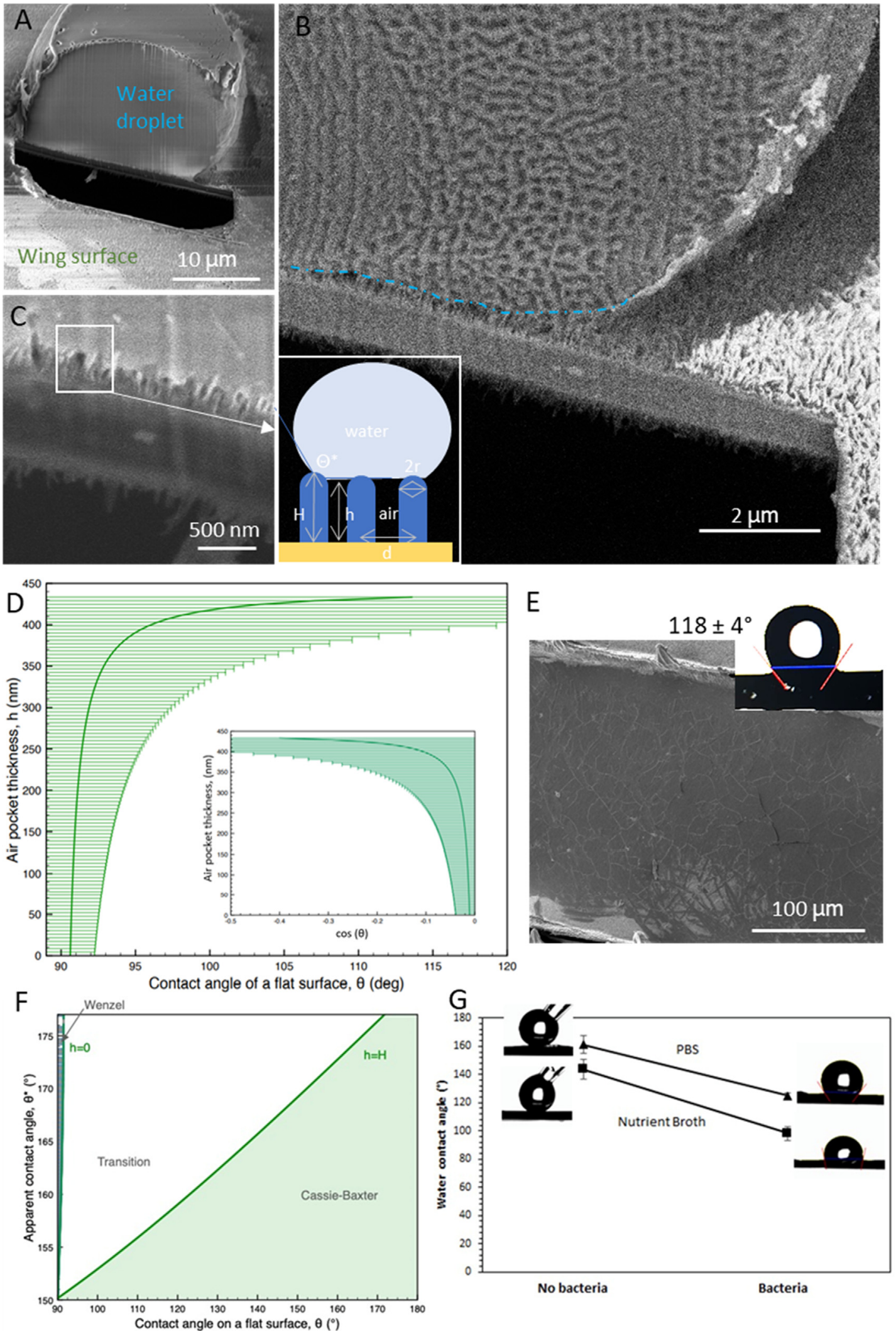

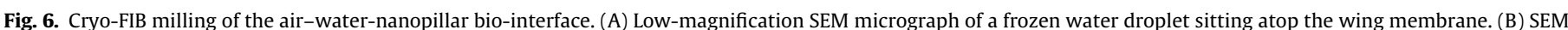

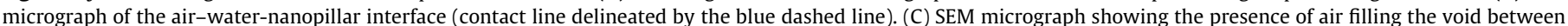

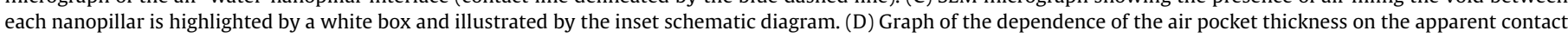

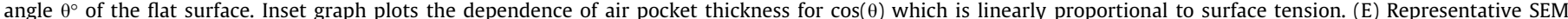

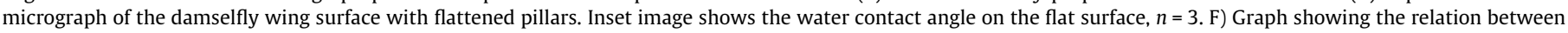

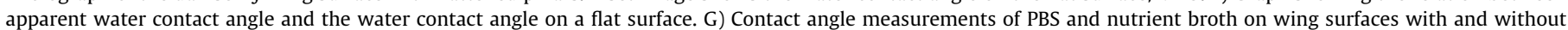
bacterial growth for $24 \mathrm{~h}, n=3$. (For interpretation of the references to colour in this figure legend, the reader is referred to the web version of this article.) 
$\Delta p=-\left(\gamma_{s l}-\gamma_{s v}\right) \frac{2 \pi r}{d^{2}-\pi r^{2}}$

or using the Young-Dupré equation for the contact angle on a flat surface (in this case it corresponds to flattened pillars to simulate a wax surface layer with no protrusions (Fig. 6E))

$\gamma_{s v}-\gamma_{s l}=\gamma_{l v} \cos \theta$

$\Delta p=\gamma_{l v} \cos \theta \frac{2 \pi r}{d^{2}-\pi r^{2}}$

or in a dimensionless form

$\Delta p \equiv \frac{r \Delta p}{\gamma_{l v}}=\frac{2 \cos \theta}{\frac{d^{2}}{\pi r^{2}}-1}$

The contact angle of a flat surface $\theta$ is related to the apparent contact angle $\theta^{*}$ on a nanostructured surface via $\cos \theta^{*}=\Phi_{s l} \cos \theta-\Phi_{l v}$, where $\Phi_{s l}$ and $\Phi_{l v}$ are the corresponding area fractions of solid liquid and liquid air interfaces with respect to the projections: $\Phi_{s l}=\left(2 \pi r(H-h)+\pi r^{2}\right) / d^{2} \quad$ and $\Phi_{l v}=\left(d^{2}-\pi r^{2}\right) / d^{2}$. Here, $h$ is the height of the interface from the bottom of the pillars. Thus,

$\cos \theta^{*}=\frac{2 \pi r(H-h)+\pi r^{2}}{d^{2}} \cos \theta-\left(1-\frac{\pi r^{2}}{d^{2}}\right)$

Since the geometrical parameters of the wing surface are known (Fig. 2A), the relationship between apparent contact angle and the contact angle on a flat surface can be shown as a diagram (Fig. 6F). The region below the curve $h=H$ corresponds to strict Cassie-Baxter regime, relating to dry wings. The experimental results confirm the water contact angle of the 'flat' surface (corresponding to the flattened nanopillar topography) to be $118 \pm 4^{\circ}$. At $120^{\circ}$, the air pocket thickness is theoretically proposed to be between 400 and $450 \mathrm{~nm}$. This measurement correlates to the condition of non-wetting; the pillars are more hydrophobic than the air and therefore the air pocket should occupy all space between the surface pillars. This can be demonstrated in the cryo-FIB cross-sectional analysis (Fig. 6A-C); liquid does not penetrate the pockets between the nanostructures, and it is only in contact with the tips of the pillars. The region between $0<h<H$ corresponds to the transition region to Wenzel regime with a partial wetting of the pillars of the wing, while the region delineated by $h=0$ and angles $\theta^{*}<150^{\circ}$ correspond to full wetting in Wenzel regime.

The pressure in the air pocket reads:

$\Delta p=\gamma_{l v} \frac{1+\cos \theta^{*}-\frac{\pi r^{2}}{d^{2}}}{\frac{2 \pi r(H-h)}{d^{2}}+\frac{\pi r^{2}}{d^{2}}} \times \frac{2 \pi r}{d^{2}\left(1-\frac{\pi r^{2}}{d^{2}}\right)}$

This expression in plotted in Figure S9 and shows how the pressure is decreasing for the small radius $r$ pillars of increasing height $H$ at a dense packing on the surface $(r \ll d)$. For example, when height of pillar is equal to its radius $H / r=1$, the pressure is augmented by $5 \%$ as compared with the liquid-vapor surface tension $\gamma_{\text {lve }}$

Surface tension governs the behavior at the interface between wing, air, and water. Furthermore, surface tension on the wings can be affected by temperature $T$ as $\sim 1 / T$. It was shown that cicada wing exhibits a 5-fold difference in temperature diffusivity in the plane of the wing as compared with across the wing [44]. The main reason being the contribution of air between nanoneedles which has temperature diffusivity of $2.2 \times 10^{-5} \mathrm{~m}^{2} / \mathrm{s}$ as compared with the wing's temperature diffusivity of 0.7 (across) and 3.6 (in-plane) $\times 10^{-7} \mathrm{~m}^{2} / \mathrm{s}$, while water has diffusivity of $1.4 \times 10^{-7} \mathrm{~m}^{2} / \mathrm{s}$ at normal conditions. Hence, the air pockets trapped on the surface of the wing are important in temperature equilibration on the surface when non-uniformity of temperature distribution might occur, and surface tension is locally modified. This is also a factor for stability of the air entrapment at the wing surface.

\section{Conclusions}

In summary, the nanopillar array on the surface of insect wings are known to play a role in mechanically inactivating microbial cells that encounter the surface $[8,20]$, however, the antifungal properties of insect wings, and the role played by the surface nanotopography, was not previously studied and understood. In this study, we confirmed the mechanism of antifungal action of superhydrophobic damselfly wings. It was demonstrated that the filamentous fungi $A$. brasiliensis and $E$. nigrum were unable to attach to damselfly wings in submerged conditions, even following one week of incubation. The prevention of fungal conidia attachment was linked to the presence of a uniquely stable air layer on both the micro- and nanoscales. These layers include the thicker micro-scale air cushion that is visible to the naked eye and the nanobubbles that exist on top (and in between) the nanopillars. Comprehensive theoretical and experimental analyses of the wing wetting states confirmed the true Cassie-Baxter wetting state of the wing surfaces. Thus, during submersion, only the tips of the wax nanopillars on the wing epicuticle were exposed to the inoculating liquid and the air pocket prevents attachment by fungal conidia. During the removal of the wing surfaces from the inoculating medium, the liquid slides off together with the fungi. Finally, we demonstrated that the antifungal and antibacterial properties of insect wing surfaces stem from two separate mechanisms that can both be attributed to the array of nanopillars found on the epicuticle of the wing membrane. While insect wings avoid bacterial colonisation by exploiting the nanopillar topology of the epicuticle surface to mechanically lyse all bacteria encountering the surface, the antifungal properties of the insect wings are governed by the ability of the surface to maintain a consistent and impenetrable air layer to repel fungal conidia, as confirmed by a comprehensive analysis and visualisation of the air-liquid interface. Since one route of fungal fouling of surfaces derives from spores and conidia being suspended in water, the minimisation of contact between water and the solid surface may be an effective tool in preventing the biofouling of abiotic surfaces. As demonstrated in this study, this can be achieved by the maintenance of a stable air film between the solid surface and the liquid, even in applications of extended water immersion, facilitated by surface nanotopography.

\section{Declaration of Competing Interest}

The authors declare that they have no known competing financial interests or personal relationships that could have appeared to influence the work reported in this paper.

\section{Acknowledgments}

The authors acknowledge ANSTO and the Australian Synchrotron for use of the IR beamline. Funding from the Australian Research Council (ARC) Industrial Transformation Research Hubs Scheme (ARC Research Hub for Australian Steel Manufacturing, Project Number IH130100017) and ARC Industrial Transformation Training Centre (ITTC) scheme (Project number IC180100005) are gratefully acknowledged. The authors acknowledge the technical services of the RMIT microscopy and microanalysis facility (RMMF), and the use of instruments and assistance at the Monash Ramaciotti Centre for Cryo-Electron Microscopy, a Node of Microscopy Australia. 


\section{Author contributions}

E.P.I, D.P.L, and S.J. conceived the study and designed the experiments. E.P.I supervised the whole work. A.A.M, P.L and D.P.L measured the antibiofouling property of the wings. A.A.M performed PCR. S.H.N contributed to the contact angle experiments with specialized techniques. D.P.L, R.C, E.H, V.K.T, G.G, G.R carried out the cryo experiments. V.B and S.J provided computational analyses of air entrapment. D.P.L wrote the paper. All authors contributed to editing and approved the final manuscript.

\section{Data and materials availability}

All data needed to evaluate the conclusions in the paper are present in the paper and/or the Supplementary Materials. Additional data related to this paper may be requested from the authors.

\section{Appendix A. Supplementary material}

Supplementary data to this article can be found online at https://doi.org/10.1016/j.jcis.2021.06.093.

\section{References}

[1] D. Byun, J. Hong, J.H. Saputra, Y.J. Ko, H.C. Lee, B.-K. Park, J.R.L. Byun, Wetting Characteristics of Insect Wing Surfaces, J. Bionic Eng. 6 (2009) 63-70.

[2] T. Wagner, C. Neinhuis, W. Barthlott, Wettability and Contaminability of Insect Wings as a Function of Their Surface Sculptures, Acta Zool. 77 (1996) 213-225.

[3] D.E. Mainwaring, S.H. Nguyen, H. Webb, T. Jakubov, M. Tobin, R.N. Lamb, A.H.F. Wu, R. Marchant, R.J. Crawford, E.P. Ivanova, The nature of inherent bactericidal activity: insights from the nanotopology of three species of dragonfly, Nanoscale 8 (2016) 6527-6534.

[4] A. Merlen, P. Brunet, Impact of Drops on Non-wetting Biomimetic Surfaces, J. Bionic Eng. 6 (2009) 330-334.

[5] S. Latthe, C. Terashima, K. Nakata, A. Fujishima, Superhydrophobic Surfaces Developed by Mimicking Hierarchical Surface Morphology of Lotus Leaf, Molecules 19 (2014) 4256-4283.

[6] X. Gao, Z. Guo, Biomimetic superhydrophobic surfaces with transition metals and their oxides: A review, J. Bionic Eng. 14 (2017) 401-439.

[7] V.K. Truong, N.M. Geeganagamage, V.A. Baulin, J. Vongsvivut, M.J. Tobin, P. Luque, R.J. Crawford, E.P. Ivanova, The susceptibility of Staphylococcus aureus CIP 65.8 and Pseudomonas aeruginosa ATCC 9721 cells to the bactericidal action of nanostructured Calopteryx haemorrhoidalis damselfly wing surfaces, Appl. Microbiol. Biotechnol. (2017) 1-8.

[8] E.P. Ivanova, J. Hasan, H.K. Webb, V.K. Truong, G.S. Watson, J.A. Watson, V.A. Baulin, S. Pogodin, J.Y. Wang, M.J. Tobin, C. Löbbe, R.J. Crawford, Natural Bactericidal Surfaces: Mechanical Rupture of Pseudomonas aeruginosa Cells by Cicada Wings, Small 8 (2012) 2489-2494.

[9] S. Pogodin, J. Hasan, V.A. Baulin, H.K. Webb, V.K. Truong, T.H. Phong, V.B. Nguyen, C.J. Fluke, G.S. Watson, J.A. Watson, J. Russell, Biophysical Model of Bacterial Cell Interactions with Nanopatterned Cicada Wing Surfaces, Biophys. J. 104 (4) (2013) 835-840.

[10] S.M. Kelleher, O. Habimana, J. Lawler, B. O’Reilly, S. Daniels, E. Casey, A. Cowley, Cicada wing surface topography: An investigation into the bactericidal properties of nanostructural features, ACS Appl. Mater. Interf. 8 (2015) $14966-14974$.

[11] L.D. Chambers, K.R. Stokes, F.C. Walsh, R.J.K. Wood, Modern approaches to marine antifouling coatings, Surf. Coat. Technol. 201 (2006) 3642-3652.

[12] M.P. Schultz, J.A. Bendick, E.R. Holm, W.M. Hertel, Economic impact of biofouling on a naval surface ship, Biofouling 27 (2010) 87-98.

[13] D.J. Giannantonio, J.C. Kurth, K.E. Kurtis, P.A. Sobecky, Effects of concrete properties and nutrients on fungal colonization and fouling, Int. Biodeterior. Biodegradation 63 (2009) 252-259.

[14] P. Guiamet, S. Borrego, P. Lavin, I. Perdomo, S.G.D. Saravia, Biofouling and biodeterioration in materials stored at the Historical Archive of the Museum of La Plata, Argentine and at the National Archive of the Republic of Cuba, Colloids Surf. B 85 (2011) 229-234.

[15] M.H. Joshi, P. Balamurugan, V.P. Venugopalan, T.S. Rao, Dense fouling in acid transfer pipelines by an acidophilic rubber degrading fungus, Biofouling 27 (2011) 621-629.
[16] S. Dobretsov, R.M.M. Abed, M. Teplitski, Mini-review: Inhibition of biofouling by marine microorganisms, Biofouling 29 (2013) 423-441.

[17] P. Forsberg, F. Nikolajeff, M. Karlsson, Cassie-Wenzel and Wenzel-Cassie transitions on immersed superhydrophobic surfaces under hydrostatic pressure, Soft Matter 7 (2011) 104-109.

[18] G. Londe, A. Chunder, A. Wesser, L. Zhai, H. Cho, Microfluidic valves based on superhydrophobic nanostructures and switchable thermosensitive surface for lab-on-a-chip (LOC) systems, Sens. Actuators B Chem. 132 (2008) 431-438.

[19] J.-W. Lee, W. Hwang, Fabrication of a superhydrophobic surface with funguscleaning properties on brazed aluminum for industrial application in heat exchangers, Appl. Surf. Sci. 442 (2018) 461-466.

[20] D.P. Linklater, V.A. Baulin, S. Juodkazis, R.J. Crawford, P. Stoodley, E.P. Ivanova, Mechano-bactericidal actions of nanostructured surfaces, Nat. Rev. Microbiol. (2020).

[21] M. Cloutier, D. Mantovani, F. Rosei, Antibacterial Coatings: Challenges, Perspectives, and Opportunities, Trends Biotechnol. 33 (2015) 637-652.

[22] X. Li, B. Wu, H. Chen, K. Nan, Y. Jin, L. Sun, B. Wang, Recent developments in smart antibacterial surfaces to inhibit biofilm formation and bacterial infections, J. Mater. Chem. B 6 (2018) 4274-4292.

[23] U. Binder, C. Lass-Flörl, Epidemiology of invasive fungal infections in the mediterranean area, Mediterr. J. Hematol. Infect. Dis. 3 (2011) e2011016.

[24] G.D. Brown, D.W. Denning, S.M. Levitz, Tackling Human Fungal Infections, Science 336 (2012). 647647.

[25] D. Outomuro, A. Torralba-Burrial, F.J. Ocharan, Distribution of the IberianCalopteryxDamselflies and Its Relation with Bioclimatic Belts: Evolutionary and Biogeographic Implications, J. Insect Sci. 10 (2010) 1-16.

[26] J. Hasan, H.K. Webb, V.K. Truong, G.S. Watson, J.A. Watson, M.J. Tobin, G. Gervinskas, S. Juodkazis, J.Y. Wang, R.J. Crawford, E.P. Ivanova, Spatial variations and temporal metastability of the self-cleaning and superhydrophobic properties of damselfly wings, Langmuir 28 (2012) 17404-17409.

[27] E. Schuster, N. Dunn-Coleman, J. Frisvad, P. van Dijck, On the safety of Aspergillus niger - a review, Appl. Microbiol. Biotechnol. 59 (4) (2002) 426435.

[28] M. Sun, G.S. Watson, Y. Zheng, J.A. Watson, A. Liang, Wetting properties on nanostructured surfaces of cicada wings, J. Exp. Biol. 212 (2009) 3148-3155.

[29] L. Dellieu, M. Sarrazin, P. Simonis, O. Deparis, J.P. Vigneron, A two-in-one superhydrophobic and anti-reflective nanodevice in the grey cicada Cicada orni (HemipteraJ, Appl. Phys. 116 (2014) 024701

[30] J.C. Valmalette, H. Raad, N. Qiu, S. Ohara, M. Capovilla, A. Robichon, Nanoarchitecture of gustatory chemosensory bristles and trachea in Drosophila wings, Sci. Rep. 5 (2015).

[31] E.B.G. Jones, Fungal adhesion, Mycolog. Res. 98 (1994) 961-981.

[32] H.A.B. Wosten, O.M.H. de Vries, J.G.H. Wessels, Interfacial Self-Assembly of a Fungal Hydrophobin into a Hydrophobic Rodlet Layer, The Plant Cell 5 (1993) 1567.

[33] H.A.B. Wösten, Hydrophobins: Multipurpose Proteins, Annu. Rev. Microbiol. 55 (2001) 625-646.

[34] S.R. Ball, A.H. Kwan, M. Sunde, Hydrophobin Rodlets on the Fungal Cell Wall, Current Topics in Microbiology and Immunology, Springer, Berlin Heidelberg, 2019.

[35] E.E. Meyer, K.J. Rosenberg, J. Israelachvili, Recent progress in understanding hydrophobic interactions, PNAS 103 (2006) 15739-15746.

[36] M. Flury, S. Aramrak, Role of air-water interfaces in colloid transport in porous media: A review, Water Resour. Res. 53 (2017) 5247-5275.

[37] B. Albijanic, O. Ozdemir, A.V. Nguyen, D. Bradshaw, A review of induction and attachment times of wetting thin films between air bubbles and particles and its relevance in the separation of particles by flotation, Adv. Colloid Interf. Sci. 159 (2010) 1-21.

[38] J. Wan, J.L. Wilson, T.L. Kieft, Influence of the Gas-Water Interface on Transport of Microorganisms through Unsaturated Porous Media, Appl. Environ. Microbiol. 60 (1994) 509-516.

[39] B.R. Dahlbeck, M. Hermansson, S. Kjelleberg, B. Norkrans, The hydrophobicity of bacteria ? An important factor in their initial adhesion at the air-water inteface, Arch. Microbiol. 128 (1981) 267-270.

[40] Y. Liu, L. Moevius, X. Xu, T. Qian, J.M. Yeomans, Z. Wang, Pancake bouncing on superhydrophobic surfaces, Nat. Phys. 10 (2014) 515-519.

[41] R. Jerdan, A. Kuśmierska, M. Petric, A.J. Spiers, Penetrating the air-liquid interface is the key to colonization and wrinkly spreader fitness, Microbiology 165 (2019) 1061-1074.

[42] T. Kajiya, F. Schellenberger, P. Papadopoulos, D. Vollmer, H.-J. Butt, 3D Imaging of Water-Drop Condensation on Hydrophobic and Hydrophilic LubricantImpregnated Surfaces, Sci. Rep. 6 (2016).

[43] A.B.D. Cassie, S. Baxter, Wettability of porous surfaces, Trans. Faraday Soc. 40 (1944) 546.

[44] J. Morikawa, M. Ryu, G. Seniutinas, A. Balčytis, K. Maximova, X. Wang, M. Zamengo, E.P. Ivanova, S. Juodkazis, Nanostructured Antireflective and Thermoisolative Cicada Wings, Langmuir 32 (2016) 4698-4703. 